\title{
छs \\ Fluctuating pancake vortices revealed by dissipation of the Josephson vortex lattice
}

\author{
A. E. Koshelev \\ Materials Science Division, Argonne National Laboratory, Argonne, Illinois 60439, USA
}

A. I. Buzdin

Institut Université de France and Université de Bordeaux, LOMA, UMR 5798, F-33405 Talence, France

I. Kakeya

Department of Electronic Science and Engineering, Kyoto University, Nishikyo, Kyoto 615-8510, Japan

\author{
T. Yamamoto* and K. Kadowaki \\ Institute of Materials Science, University of Tsukuba, Tsukuba, Ibaraki 305-8573, Japan
}

(Received 4 April 2011; published 27 June 2011)

\begin{abstract}
In strongly anisotropic layered superconductors in tilted magnetic fields, the Josephson vortex lattice coexists with the lattice of pancake vortices. Due to the interaction between them, the dissipation of the Josephson vortex lattice is very sensitive to the presence of the pancake vortices. If the $c$-axis magnetic field is smaller than the corresponding lower critical field, the pancake stacks are not formed but the individual pancakes may exist in the fluctuational regime either near the surface in large-size samples or in the central region for small-size mesas. We calculate the contribution of such fluctuating pancake vortices to the $c$-axis conductivity of the Josephson vortex lattice and compare the theoretical results with measurements on small mesas fabricated out of $\mathrm{Bi}_{2} \mathrm{Sr}_{2} \mathrm{CaCu}_{2} \mathrm{O}_{8+\delta}$ crystals. A fingerprint of fluctuating pancakes is a characteristic exponential dependence of the $c$-axis conductivity observed experimentally. Our results provide strong evidence of the existence of the fluctuating pancakes and their influence on the Josephson vortex lattice dissipation.
\end{abstract}

DOI: 10.1103/PhysRevB.83.224515

PACS number(s): 74.40.-n, 74.50.+r, 74.25.Uv, 74.20.De

\section{INTRODUCTION}

Vortex physics in layered superconductors is extremely rich and interesting. This is mostly related to the fact that the magnetic field parallel to the layers penetrates in the form of the Josephson vortices (JV's), ${ }^{1}$ while the perpendicular field creates the stacks of the pancake vortices (PV's). ${ }^{2}$ In the tilted magnetic field, the attractive interaction between JV's and PV's (Ref. 3) leads to the creation of many unusual vortex states: mixed chain-lattice states, ${ }^{4}$ JV's decorated by PV's, ${ }^{5}$ etc. (see as a review Ref. 6). The interaction between the PV and JV lattices is revealed also in their dynamic properties. The dynamics of the JV lattice in $\mathrm{Bi}_{2} \mathrm{Sr}_{2} \mathrm{CaCu}_{2} \mathrm{O}_{8+\delta}(\mathrm{Bi} 2212)$ mesas has been explored by several experimental groups. ${ }^{7-12}$ Due to the weak intrinsic dissipation, the friction force acting on the moving JV lattice is small. That is why even the presence of a small amount of the PV stacks strongly affects the dynamic of the JV's. The physical reason is that the moving JV's induce displacements of the PV's, which have large viscous friction due to the normal cores. This strongly enhances the JV friction, leading to a decrease of the measured $c$-axis resistivity of the sample. Recently, this effect has been studied in detail in Refs. 13 and 14. To the best of our knowledge, this is the only known situation in which adding vortices to a superconductor reduces its resistivity. Therefore, by studying the dynamic properties of the JV lattice, we may obtain the information about the presence of the PV's.

In the present paper, we use this tool to study the penetration of the PV's in superconducting mesas of the Bi2212 intrinsic Josephson junctions. If the magnetic field is applied exactly along the layers, then the $c$-axis resistivity is governed by the friction force acting on the moving JV lattice. When the perpendicular component of magnetic field $H_{z}$ is switched on, the PV stack can enter into the sample when $H_{z}$ reaches the value of the perpendicular low critical field $H_{c 1}^{c}{ }^{15}$

The $c$-axis field penetration is rather special in the case of layered superconductors with weak Josephson coupling between the layers. Indeed, the field starts to penetrate in the form of the PV's created near the surface in the fluctuational regime. The energy of a single PV near the surface has been calculated in Ref. 16 (see also Ref. 17), and above a certain field (somewhat lower than $H_{c 1}^{c}$ ) it has a minimum at a distance of the order of the London penetration depth $\lambda$ from the surface. In small-size samples with lateral dimensions comparable with the London penetration depth, this energy minimum appears in the center of the sample. The concentration of the fluctuation PV's is determined by this minimum energy, which, in turn, depends on the perpendicular magnetic field.

The presence of these fluctuational PV's increases the friction of the JV's, and then it may be directly monitored by the measurement of the $c$-axis resistivity. We obtain a very characteristic exponential dependence of the $c$-axis conductivity versus $H_{z}$, reflecting the varying density of the thermally activated PV's near the surface or in the center of small samples. This dependence is clearly observed experimentally. These results demonstrate that the JV's serve as a perfect tool to detect the presence of the PV's in the fluctuational regime.

The behavior of the JV lattice has special features in the mesas with lateral sizes of a few Josephson lengths used in our experiments. In this case, bulk interactions favoring the triangular lattice compete with edge interactions favoring the rectangular lattice. Due to this competition, the JV lattice 
undergoes a series of structural phase transitions between the triangular and rectangular configurations. ${ }^{18,19}$ The regions of rectangular lattice dominate at large fields. Because the inplane current is absent in the rectangular lattice, its interaction with PV's is very weak. Therefore, the fluctuational PV's have a strong influence only on dissipation of the triangular lattice. Due to commensurability effects, the $c$-axis resistivity has pronounced oscillations as a function of the magnetic field. ${ }^{10}$ The period of these oscillations changes from half flux quantum per junction at small fields to one flux quantum per junction at large fields, ${ }^{11,12}$ and the crossover field between these regimes is proportional to the mesa width. ${ }^{19}$

The paper is organized as follows. In Sec. II, we describe the theory of the effect. In particular, in Sec. II A we derive the general formalism describing the response of the statistical ensemble of PV's in the static potential (created by interaction with the surface) to the moving periodic potential (due to the interaction with moving JV lattice). We provide the expression for the resulting dissipation. In Sec. II B 1, we detail the potential of the PV interaction with the surface in the presence of the perpendicular field $H_{z}$. We also consider the potential energy of a PV in finite-size samples with simple geometries (strip and cylinder). The energy of the interaction between the PV located near the surface and the dense lattice of the JV's is calculated in Sec. II C. We analyze the general expression for the PV contribution to the JV lattice conductivity in Sec. II D. In Sec. III, we present the experimental data on the $c$-axis conductivity of $\mathrm{Bi}_{2} \mathrm{Sr}_{2} \mathrm{CaCu}_{2} \mathrm{O}_{8+\delta}$ mesas with different lateral sizes in tilted magnetic field and compare the results with theoretical predictions.

\section{THEORY}

\section{A. Fluctuation pancake vortices in static and moving periodic potentials: General consideration}

We consider the geometry with the parallel-to-layers magnetic field directed along the $x$ axis $H_{\|}=H_{x}$ and, therefore, the perpendicular current (along the $c$ axis) will drive the JV lattice along the $y$ axis; see Fig. 1(a). The PV located at distance $x$ will interact with the surface ( $y z$ plane) and with a current screening the perpendicular field $H_{z}$ as well as with a moving JV lattice. The first two contributions are described by the static potential $U_{0}(x)$. The moving periodic potentials of the JV lattice is $\tilde{U}(x) \cos (k y-\omega t)$, corresponding to the velocity $v=\omega / k$. This motion generates an electric field $E_{z}=H_{x} v / c$. Therefore, we are interested in the behavior of the PV's in the two-dimensional potential

$$
U(\mathbf{r}, t)=U_{0}(x)+\tilde{U}(x) \cos (k y-\omega t) .
$$

We start from a general consideration of a model in which the PV's are considered as an ensemble of particles with concentration $n$ in the external time-dependent potential $U(\mathbf{r}, t)$. The particle current $\mathbf{j}$ (Ref. 20) and particle-conservation equation are given by

$$
\begin{gathered}
\mathbf{j}=-D\left(\nabla n+\frac{\nabla U}{T} n\right), \\
\frac{\partial n}{\partial t}-D \nabla\left(\nabla n+\frac{\nabla U}{T} n\right)=0,
\end{gathered}
$$

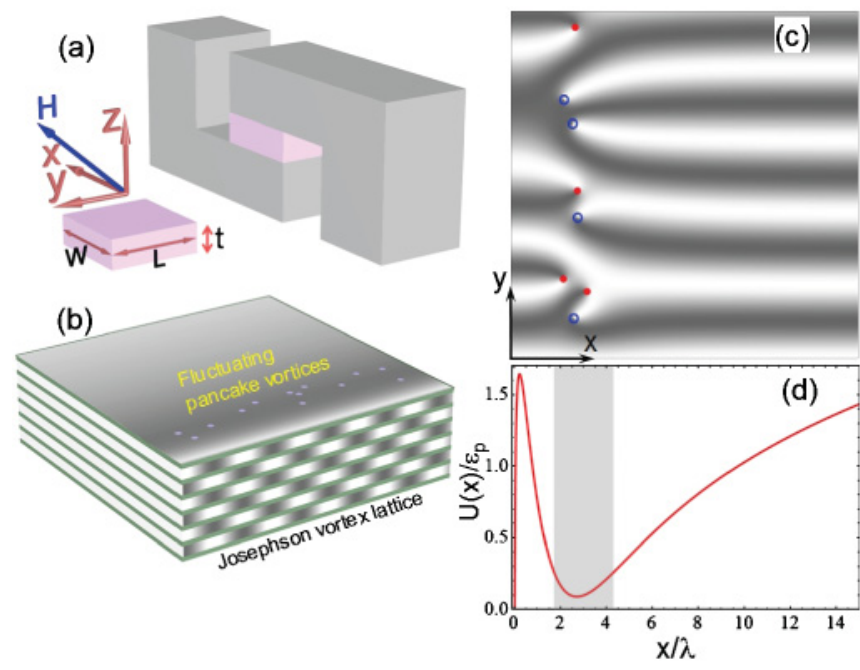

FIG. 1. (Color online) These schematic figures illustrate mesa geometry and the interaction between the dense JV lattice and fluctuational PV's near the surface. (a) The right part shows the outline of the structure fabricated from Bi2212 crystal to study $c$-axis transport through the highlighted mesa region. The left part shows definitions of axes and mesa sizes. (b) Three-dimensional sketch of the JV lattice inside the junctions (front face) and the fluctuating PVs near the surface (top face). (c) The gray-level plot of the cosine of the phase difference between two neighboring layers that have periodic modulation due to the JV lattice and perturbations due to randomly located PV's in two layers shown by open and closed circles. (d) The energy profile of PV's near the surface in the $c$-axis magnetic field close to the lower-critical field. The PV density is enhanced near the potential minimum. This highlighted region near the minimum of the potential gives a dominating contribution to dissipation of the JV lattice.

where $D$ is the diffusion constant. These equations describe the particle density and current within a single layer and contain only the in-plane gradients.

In the absence of the moving potential, the particle current is naturally equal to zero and the equilibrium density $n_{0}(x) \propto$ $\exp \left[-U_{0}(x) / T\right] .{ }^{21}$ Introducing the perturbation for the density $v(\mathbf{r}, t)$ and current due to the moving potential,

$$
\begin{aligned}
& n(\mathbf{r}, t)=n_{0}(x)[1+v(\mathbf{r}, t)], \\
& \tilde{\mathbf{j}}=-D\left(\nabla v+\frac{\nabla \tilde{U}}{T}\right) n_{0},
\end{aligned}
$$

we have the following equation for the small perturbation:

$$
\frac{\partial v}{\partial t} n_{0}-D \nabla\left(n_{0} \nabla v\right)=\frac{D}{T} \nabla\left(n_{0} \nabla \tilde{U}\right) .
$$

Using the complex presentation

$$
\begin{aligned}
\tilde{U}(\mathbf{r}, t) & =\tilde{U}(x) \operatorname{Re}\{\exp [i(k y-\omega t)]\}, \\
v(\mathbf{r}, t) & =\operatorname{Re}\left\{v_{\omega}(x) \exp [i(k y-\omega t)]\right\},
\end{aligned}
$$

we obtain the equation for the complex amplitude $v_{\omega}(x)$,

$$
\begin{gathered}
\left(-i \eta \omega+T k^{2}\right) v_{\omega}-\frac{T}{n_{0}} \nabla_{x}\left(n_{0} \nabla_{x} v_{\omega}\right), \\
=\frac{1}{n_{0}} \nabla_{x}\left(n_{0} \nabla_{x} \tilde{U}\right)-k^{2} \tilde{U}
\end{gathered}
$$

where $\eta=T / D$ is the viscosity coefficient. 
Assuming that the frequency is small, we solve this equation by iterations. The first iteration corresponds to quasiequilibrium,

$$
v_{\omega}^{(0)}=-\frac{\tilde{U}}{T}, \quad \mathbf{j}^{(0)}=0 .
$$

The next iteration has to be found from the equation

$$
D\left(\frac{1}{n_{0}} \nabla_{x}\left(n_{0} \nabla_{x} v_{\omega}^{(1)}\right)-k^{2} v_{\omega}^{(1)}\right)=i \omega \frac{\tilde{U}}{T} .
$$

Near the minimum of the potential, the equilibrium density can be represented as

$$
\begin{aligned}
& n_{0}(x)=n_{00} \exp \left[-\frac{K x^{2}}{2 T}\right], \\
& n_{00}=n_{\xi} \exp \left(-U_{\min } / T\right) .
\end{aligned}
$$

We also assume that the typical scale of $\tilde{U}(x)$ variation is larger than the confining length of the static potential $\sqrt{T / K}$ so that we can use the expansion near the minimum, $\tilde{U}(x) \approx$ $\tilde{U}_{0}-\tilde{F} x$. In this case, Eq. (7) has a simple analytical solution,

$$
v_{\omega}^{(1)}=-\frac{i \omega \tilde{U}_{0}}{D k^{2} T}+\frac{i \omega \tilde{F} x}{D\left(K+T k^{2}\right)},
$$

which gives the oscillating particle-current amplitudes

$$
\begin{gathered}
\tilde{j}_{x}=-\frac{i \omega \tilde{F}}{K+T k^{2}} n_{0}, \\
\tilde{j}_{y}=i k \frac{i \omega \tilde{U}}{T k^{2}} n_{0} .
\end{gathered}
$$

The energy dissipation in steady state can be evaluated as

$$
W=-\int d x\langle\mathbf{j} \nabla U\rangle=\eta \int d x\left\langle\frac{\mathbf{j}^{2}}{n}\right\rangle \approx \frac{\eta}{2} \int d x \frac{\left|j_{x}\right|^{2}+\left|j_{y}\right|^{2}}{n_{0}} .
$$

Substituting currents (9a) and (9b), we finally obtain

$$
\begin{aligned}
W & =\frac{\eta \omega^{2}}{2}\left(\frac{\tilde{F}^{2}}{\left(K+T k^{2}\right)^{2}}+\frac{\tilde{U}^{2}}{T^{2} k^{2}}\right) \int d x n_{0}(x) \\
& \approx \frac{\eta \omega^{2}}{2} n_{\xi} \exp \left(-\frac{U_{\min }}{T}\right) \sqrt{\frac{2 \pi T}{K}}\left(\frac{\tilde{F}^{2}}{\left(K+T k^{2}\right)^{2}}+\frac{\tilde{U}^{2}}{T^{2} k^{2}}\right) .
\end{aligned}
$$

This formula determines energy losses caused by an arbitrary moving periodic potential near the minimum of the static potential. We will use it to evaluate the contribution of the fluctuating PV's to the JV lattice conductivity. For this, in the following sections, we will obtain explicit expressions for the static and dynamic potentials.

\section{B. Energy of the pancake vortex}

\section{Energy profile of the pancake vortex near the surface in large-size samples}

If the perpendicular magnetic field $H_{z}$ is slightly lower than $H_{c 1}^{c}$, the PV stacks (Abrikosov vortices) do not penetrate into the sample, but the PV's can exist near the surface at the fluctuation regime. The energy of the pancake vortex at the distance $x$ from the surface is given by ${ }^{16}$

$$
\begin{aligned}
U_{0}(x) \approx & \frac{s \Phi_{0}^{2}}{(4 \pi \lambda)^{2}} \ln \frac{x}{\xi}-\frac{s \Phi_{0} H_{z}}{4 \pi}\left[1-\exp \left(-\frac{x}{\lambda}\right)\right] \\
= & \frac{s \Phi_{0}\left(H_{c 1}^{c}-H_{z}\right)}{4 \pi}+\frac{s \Phi_{0}^{2}}{(4 \pi \lambda)^{2}} \ln \frac{x}{\lambda} \\
& +\frac{s \Phi_{0} H_{z}}{4 \pi} \exp \left(-\frac{x}{\lambda}\right),
\end{aligned}
$$

where $s$ is the distance between superconducting layers and $H_{c 1}^{c} \approx\left[\Phi_{0} /\left(4 \pi \lambda^{2}\right)\right][\ln (\lambda / \xi)+0.5]$ is the lower critical field. Above a certain field, this potential will have a minimum at distance $\sim \lambda$ from the surface; see the schematic in Fig. 1(d). Taking the derivative,

$$
\frac{d U_{0}}{d x}=\frac{s \Phi_{0}}{4 \pi \lambda}\left[\frac{\Phi_{0}}{4 \pi \lambda^{2}} \frac{\lambda}{x}-H_{z} \exp \left(-\frac{x}{\lambda}\right)\right],
$$

we find the condition for the minimum,

$$
u \exp (-u)=\frac{\Phi_{0}}{4 \pi \lambda^{2} H_{z}},
$$

with $u=x / \lambda$. The minimum exists if

$$
H_{z}>H_{s}=\frac{e \Phi_{0}}{4 \pi \lambda^{2}} .
$$

Above this field, the pancake energy at the minimum is determined by the equations

$$
\begin{gathered}
U_{\min }=\frac{s \Phi_{0}}{4 \pi}\left(H_{c 1}^{c}-H_{z}\right)+\varepsilon_{p}\left[\ln \left(u_{\min }\right)+\frac{1}{u_{\min }}\right], \\
u_{\min } \exp \left(-u_{\min }\right)=\frac{\Phi_{0}}{4 \pi \lambda^{2} H_{z}},
\end{gathered}
$$

where $\varepsilon_{p}=s \Phi_{0}^{2} /(4 \pi \lambda)^{2}$ is the PV energy scale. As $u_{\min }$ roughly behaves as $\ln \left(4 \pi \lambda^{2} H_{z} / \Phi_{0}\right)$, the main dependence of $U_{\min }$ on $H_{z}$ is given by the first term, i.e., the minimum energy approximately linearly decreases with the field. For Bi2212, $s \approx 1.56 \mathrm{~nm}$ and we estimate the slope of this dependence as $d U_{\min } / d H_{z} \approx-18.6 \mathrm{~K} / \mathrm{G}$.

Expanding the potential (12) near the minimum, $U(x)=$ $U_{\min }+K\left(x-x_{\min }\right)^{2} / 2$, we evaluate the spring constant $K$ as

$$
\begin{gathered}
K=\frac{d^{2} U}{d x^{2}}=\frac{\varepsilon_{p}}{\lambda^{2}} a_{K}, \\
a_{K}=\frac{1}{u_{\min }}-\frac{1}{u_{\min }^{2}} \approx \frac{1}{\ln h},
\end{gathered}
$$

with $h=4 \pi \lambda^{2} H_{z} / \Phi_{0}$.

The condition of the vortex line formation is roughly $U_{\min }=$ 0 . In such a case, the pancakes will accumulate near the surface and form a vortex line. The magnetic field $h_{1}$ at which this happens is determined by the equations

$$
u_{1}=\ln h_{1}-\ln u_{1}, \quad h_{1}=\frac{\ln \left(\kappa u_{1}\right)}{1-\exp \left(-u_{1}\right)},
$$

which can be solved by the iterations

$$
u_{1} \approx \ln h_{1}-\ln \ln h_{1}, \quad h_{1} \approx \ln \kappa+\ln [\ln (\ln \kappa)] .
$$

Therefore, this field is only slightly larger than $H_{c 1}^{c}$. The Bean-Livingston barrier in layered superconductors with 
high anisotropy ratio is eliminated at sufficiently high temperatures. ${ }^{22}$ In the field interval $H_{s}<H_{z}<H_{c 1}^{c}$, we may expect the accumulation of the fluctuating PV's near the surface, and their density would increase dramatically when $H_{z}$ approaches $H_{c 1}^{c}$.

\section{Energy of the pancake vortex in small-size mesas}

The previous analysis considered the case of the samples much larger than the London penetration depth $\lambda$. When their dimensions are comparable with $\lambda$ (which may be the case for the small mesas), the minima of the PV's energy near the surfaces merge and the dependence (14) changes. We may illustrate such a crossover in the case of the strip of the width $w$. Using the results of Ref. 23, in which the vortex energy in the narrow superconducting strip was calculated, we write the expression for the energy of the pancake at the distance $x$ from the strip edge as

$$
\begin{aligned}
U_{0}(x)= & \frac{s \Phi_{0}\left(H_{c 1}^{c}-H_{z}\right)}{4 \pi}+\frac{s \Phi_{0}^{2}}{(4 \pi \lambda)^{2}} \ln \left[\frac{w}{\pi \lambda} \sin \left(\frac{\pi x}{w}\right)\right] \\
& +\frac{s \Phi_{0} H_{z}}{4 \pi} \frac{\cosh \left(\frac{x-w / 2}{\lambda}\right)}{\cosh \left(\frac{w}{2 \lambda}\right)} .
\end{aligned}
$$

In contrast to wide strips, which have two energy minima near the edges, the energy of the narrow strip at high fields has only one minimum at the strip center. Two minima merge in the center when the magnetic field reaches the typical value $H_{s 0}$,

$$
H_{s 0}=\frac{\pi \Phi_{0}}{4 w^{2}} \cosh \left(\frac{w}{2 \lambda}\right),
$$

and above this field the minimum energy is given by its value at $x=w / 2$,

$$
\begin{aligned}
U_{\min }= & \frac{s \Phi_{0}}{4 \pi}\left[H_{c 1}^{c}-H_{z}\left(1-\frac{1}{\cosh (w / 2 \lambda)}\right)\right] \\
& +\frac{s \Phi_{0}^{2}}{(4 \pi \lambda)^{2}} \ln \left(\frac{w}{\pi \lambda}\right) .
\end{aligned}
$$

At the field

$$
H_{s 1} \approx \frac{\Phi_{0}}{4 \pi \lambda^{2}}\left[\ln \left(\frac{w}{\pi \xi}\right)+0.5\right] \frac{\cosh (w / 2 \lambda)}{\cosh (w / 2 \lambda)-1},
$$

$U_{\min }$ reaches zero corresponding to the condition of fast stack formation in the center. This field is slightly larger than the lower critical field for the strip, $H_{c 1}^{\text {st }}$, which is determined by the energy of the complete PV stack at the center. In particular, for narrow strips $(w<\lambda), H_{s 1} \approx$ $H_{c 1}^{\text {st }} \approx\left[2 \Phi_{0} /\left(\pi w^{2}\right)\right]\{\ln [w /(\pi \xi)]+0.5\}$; see, e.g., Ref. 24 . The formation of the PV stacks starts at the center when the field $H_{s 0}$ becomes smaller than $H_{c 1}^{\text {st }}$, giving the approximate condition

$$
\left[\cosh \left(\frac{w}{2 \lambda}\right)-1\right] \frac{\pi^{2} \lambda^{2}}{w^{2}}<\ln \kappa+0.5
$$

For $\kappa \approx 100$, this happens already for rather wide strips, $w \lesssim 9 \lambda$. Note that at this value, the finite-size correction to the penetration field is small, $H_{c 1}^{\mathrm{st}} \approx H_{c 1}^{c}$.

Comparing the field dependences (14) and (18), we see that in the narrow strips, the slope of the $U_{\min }$ versus $H_{z}$ dependence should be smaller by the geometrical factor
$[1-1 / \cosh (w / 2 \lambda)]<1$. Due to the temperature dependence of $\lambda$, we may expect to observe the crossover between these regimes for the strips with $w \gtrsim \lambda(0)$ by varying the temperature. Note also that when the minimum $U_{0}(x)$ just appears at the center of the strip at $H_{z}=H_{s 0}$, the spring constant $K$ vanishes.

For mesas small in both the $x$ and $y$ directions, the geometrical factor will be different, but a qualitative trend is expected to be the same. To illustrate this case, we consider the cylindrical geometry, which can also be treated analytically. The energy of the pancake vortex at distance $\rho$ from the center of the mesa with radius $R$ may be calculated in the same way as the energy of the vortex in a small superconducting disk, using an image method. ${ }^{25}$ In the result, we have

$$
\begin{aligned}
U_{0}(\rho)= & \frac{s \Phi_{0}\left(H_{c 1}^{c}-H_{z}\right)}{4 \pi}+\frac{s \Phi_{0}^{2}}{(4 \pi \lambda)^{2}} \ln \left(\frac{R^{2}-\rho^{2}}{R \lambda}\right) \\
& +\frac{s \Phi_{0} H_{z}}{4 \pi} \frac{I_{0}(\rho / \lambda)}{I_{0}(R / \lambda)},
\end{aligned}
$$

where $I_{0}(z)$ is the modified Bessel function. The minimum energy for the fluctuating pancakes is shifted to the center of the mesa if the field is sufficiently large. With the help of expression (20), we find that it occurs at fields

$$
H_{z}>H_{s 0}^{\mathrm{cyl}}=\frac{\Phi_{0}}{\pi R^{2}} I_{0}\left(\frac{R}{\lambda}\right) .
$$

The lower critical field of the cylinder $H_{c 1}^{\mathrm{cyl}}$ was calculated in Ref. 26,

$$
H_{c 1}^{\mathrm{cyl}}=\frac{I_{0}(R / \lambda)}{I_{0}(R / \lambda)-1} \frac{\Phi_{0}}{4 \pi \lambda^{2}}\left[\ln \kappa+0.5-\frac{K_{0}(R / \lambda)}{I_{0}(R / \lambda)}\right] .
$$

In the limit $R \ll \lambda$, the lower critical field is $H_{c 1}^{\text {cyl }}=$ $\left[\Phi_{0} /\left(\pi R^{2}\right)\right][\ln (R / \xi)+0.38]$ and the regime of the accumulation of the fluctuating pancakes at the center of the mesa is always realized when the field $H_{z}$ starts to approach $H_{c 1}^{\text {cyl }}$. For $\kappa \approx 100$, the condition $H_{s 0}^{\text {cyl }}<H_{c 1}^{\text {cyl }}$ corresponds to the mesas with $R \lesssim 5.3 \lambda$. Note that the numerical value of the critical diameter $2 R \approx 10.6 \lambda$ is rather close to the critical width $w \approx 9 \lambda$ for the strip geometry. For smaller mesas at fields just below $H_{c 1}^{\text {cyl }}$, the minimum energy is realized for the $\mathrm{PV}$ located in the center,

$$
\begin{aligned}
U_{\min }= & U_{0}(0)=\frac{s \Phi_{0} H_{c 1}^{c}}{4 \pi}+\frac{s \Phi_{0}^{2}}{(4 \pi \lambda)^{2}} \ln \left(\frac{R}{\lambda}\right) \\
& -\frac{s \Phi_{0} H_{z}}{4 \pi}\left[1-\frac{1}{I_{0}(R / \lambda)}\right],
\end{aligned}
$$

meaning that the slope of the $U_{\min }$ versus $H_{z}$ dependence in this regime should be smaller by the factor $\left[1-1 / I_{0}(R / \lambda)\right]$, comparing to the large mesas. This slope again may substantially vary with temperature due to the temperature dependence of $\lambda$. The overall behavior is similar to the stripe case. For the cylindrical mesa with the same diameter as the stripe width, the slope reduction is close and somewhat smaller than one for the stripe. 


\section{Interaction of a pancake vortex near the surface with the dense Josephson vortex lattice}

We consider the case of the dense JV lattice when the JV's cores overlap. This situation is realized when the in-plane magnetic field $B_{\|}$is larger than the characteristic field $B_{\mathrm{cr}}=$ $\Phi_{0} /\left(2 \pi \gamma s^{2}\right)$, where the anisotropy ratio $\gamma=\lambda_{c} / \lambda$ determines the Josephson length $\lambda_{J}=\gamma s .{ }^{1}$ The interaction of the dense JV lattice with a PV-stack lattice was first considered in Ref. 27. The phase distribution for the triangular Josephson vortex lattice at this high-field regime in the presence of pancake vortices is determined by the equations

$$
\begin{array}{r}
\lambda_{J}^{2} \Delta \phi_{n}+\sin \left[\phi_{n+1}-\phi_{n}+\phi_{v, n+1}-\phi_{v, n}-k_{H}(y-v)\right] \\
\quad+\sin \left[\phi_{n}-\phi_{n-1}+\phi_{v, n}-\phi_{v, n-1}-k_{H}(y-v)\right]=0,
\end{array}
$$

where $k_{H}=2 \pi s B_{\|} / \Phi_{0}$ and $\phi_{v, n}$ are the pancake-vortex phases. The parameter $v$ describes displacement of the JV lattice. In these equations, the $\pi$ phase shift between the phase differences in the neighboring junctions is already taken into account.

We consider a single pancake in the layer $n=0$ in a largesize sample at a distance $x_{0}$ from the surface,

$$
\phi_{v, n}=\delta_{n, 0}\left(\arctan \frac{y}{x-x_{0}}-\arctan \frac{y}{x+x_{0}}\right),
$$

where the second term is the contribution from the mirror image, which is required to satisfy the condition of vanishing normal current at the surface $\partial \phi_{v, n} / \partial x=0$ for $x=0$. At high in-plane fields, the Josephson current can be treated as a small perturbation and Eq. (23) can be solved by iterations. The first iteration for the zeroth layer obeys the equation

$$
\lambda_{J}^{2} \Delta \phi_{0}^{(1)}=2 \cos \phi_{v, 0} \sin \left[k_{H}(y-v)\right],
$$

where

$$
\cos \left[\phi_{v, 0}(\mathbf{r})\right]=\frac{r^{2}-x_{0}^{2}}{\sqrt{\left(r^{2}+x_{0}^{2}\right)^{2}-4 x^{2} x_{0}^{2}}} .
$$

Without a PV, the first-order phase iteration is given by $\phi_{0}^{(1)}(\mathbf{r})=-2\left(\lambda_{J} k_{H}\right)^{-2} \sin \left[k_{H}(y-v)\right]$. The solution of Eq. (24) can be written as

$$
\begin{aligned}
\phi_{0}^{(1)}(\mathbf{r})= & -\frac{2}{\lambda_{J}^{2} k_{H}^{2}} \sin \left[k_{H}(y-v)\right]+\frac{1}{\pi \lambda_{J}^{2}} \int d \mathbf{r}^{\prime} \ln \frac{\left|\mathbf{r}-\mathbf{r}^{\prime}\right|}{r_{0}} \\
& \times\left\{\cos \left[\phi_{v, 0}\left(\mathbf{r}^{\prime}\right)\right]-1\right\} \sin \left[k_{H}\left(y^{\prime}-v\right)\right],
\end{aligned}
$$

where the integration is in the limits $-\infty<x^{\prime}, y^{\prime}<\infty$ and the region $x^{\prime}<0$ accounts for the image contribution. The force acting on the pancake is determined by the phase gradient at its position,

$$
f_{\alpha}=\frac{2 s \Phi_{0}^{2}}{(4 \pi \lambda)^{2}} e_{\alpha \beta z} \nabla_{\beta} \phi_{0}^{(1)},
$$

where $e_{\alpha \beta \gamma}$ is the Levi-Civita symbol $\left(e_{x y z}=-e_{y x z}=1\right.$ and $e_{\alpha \alpha z}=0$ ). Computing the phase gradient and taking it at the pancake position $\mathbf{r}=\left(x_{0}, 0\right)$, we obtain

$$
\nabla_{x} \phi_{0}^{(1)}\left(x_{0}, 0\right)=\frac{\sin \left(k_{H} v\right)}{\pi \lambda_{J}^{2} k_{H}} \mathcal{I}_{x}\left(k_{H} x_{0}\right),
$$

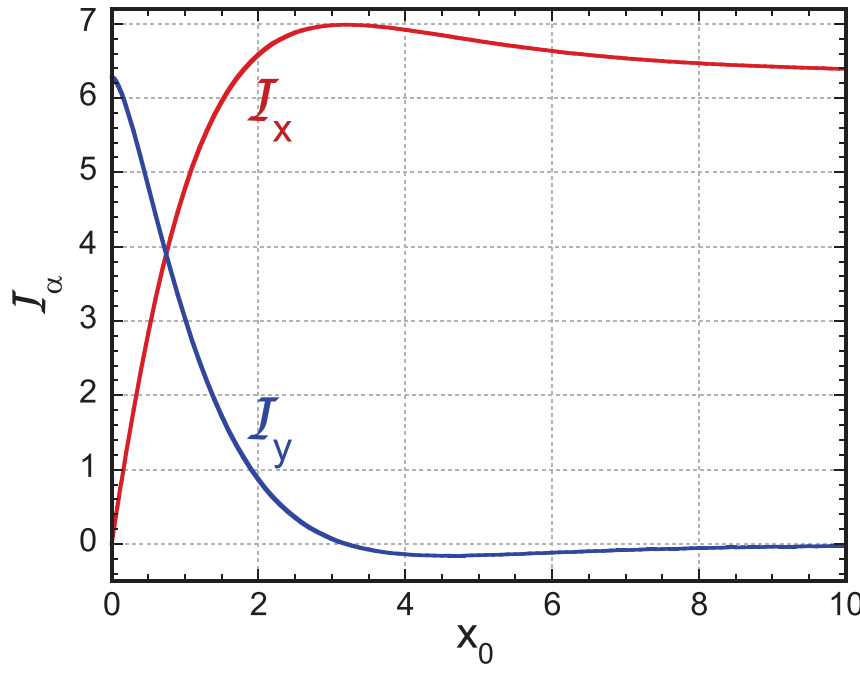

FIG. 2. (Color online) Numerically computed integrals $\mathcal{I}_{x}\left(x_{0}\right)$ and $\mathcal{I}_{y}\left(x_{0}\right)=\mathcal{I}_{x}^{\prime}\left(x_{0}\right)$ defined by Eqs. (27c) and (27d), which determine the force acting on the pancake vortex near the surface by the dense Josephson vortex lattice.

$$
\begin{gathered}
\nabla_{y} \phi_{0}^{(1)}\left(x_{0}, 0\right)=\frac{\cos \left(k_{H} v\right)}{\pi \lambda_{J}^{2} k_{H}} \mathcal{I}_{y}\left(k_{H} x_{0}\right), \\
\mathcal{I}_{x}\left(x_{0}\right)=-\int d \mathbf{r} \frac{x_{0}-x}{\left(x_{0}-x\right)^{2}+y^{2}} \frac{r^{2}-x_{0}^{2}}{\sqrt{\left(r^{2}+x_{0}^{2}\right)^{2}-4 x^{2} x_{0}^{2}}} \cos y, \\
\mathcal{I}_{y}\left(x_{0}\right)=\int d \mathbf{r} \frac{y}{\left(x_{0}-x\right)^{2}+y^{2}} \frac{r^{2}-x_{0}^{2}}{\sqrt{\left(r^{2}+x_{0}^{2}\right)^{2}-4 x^{2} x_{0}^{2}}} \sin y .
\end{gathered}
$$

These integrals have the followings asymptotics: $\mathcal{I}_{y}\left(x_{0}\right) \rightarrow$ $2 \pi, \mathcal{I}_{x}\left(x_{0}\right) \rightarrow 0$ for $x_{0} \rightarrow 0$ and $\mathcal{I}_{y}\left(x_{0}\right) \rightarrow 0, \mathcal{I}_{x}\left(x_{0}\right) \rightarrow 2 \pi$ for $x_{0} \rightarrow \infty$. Moreover, as the force components have to be derivatives of the same potential, one can demonstrate that the integrals are connected as $\mathcal{I}_{y}(x)=\mathcal{I}_{x}^{\prime}(x)$. Numerically computed integrals are plotted in Fig. 2.

Finally, the main result of this section is the expression for the potential energy of the PV in the presence of the JV lattice [compare with Eq. (1)]:

$$
\tilde{U}(\mathbf{r})=\frac{2 s \Phi_{0}^{2}}{(4 \pi \lambda)^{2}} \frac{\mathcal{I}_{x}\left(k_{H} x\right)}{\pi \lambda_{J}^{2} k_{H}^{2}} \cos \left(k_{H} y\right) .
$$

The interaction between the JV lattice and the PV near the minimum of the surface potential is determined by the reduced in-plane field,

$$
h_{\|} \equiv k_{H} \lambda=2 \pi s \lambda B_{\|} / \Phi_{0} .
$$

Taking typical values $\lambda \approx 0.3 \mu \mathrm{m}$ at $70 \mathrm{~K}$ and $B_{\|}=1 \mathrm{~T}$, we estimate $h_{\|} \approx 1.42$.

\section{Contribution of fluctuating pancake vortices near the surface to the conductivity of the Josephson vortex lattice}

Interaction with the surface pancake vortices enhances dissipation of the Josephson vortex lattice. An additional channel of dissipation makes an additional contribution to 
the JV conductivity, $\sigma_{\mathrm{JV}}=\sigma_{\mathrm{JV}}^{(0)}+\sigma_{p}$, where $\sigma_{\mathrm{JV}}^{(0)}$ is the JV conductivity in the absence of PV's and $\sigma_{p}$ is the excess conductivity due to the interaction with pancakes. The corresponding pancake surface contribution, $\sigma_{p}$, can be evaluated using the energy-dissipation formula (11),

$$
\begin{aligned}
W= & \sigma_{p} E_{z}^{2} L_{x} L_{y} s=2 \frac{\eta \omega^{2}}{2} n_{\xi} L_{y} \exp \left(-\frac{U_{\min }}{T}\right) \sqrt{\frac{2 \pi T}{K}} \\
& \times\left(\frac{\tilde{F}^{2}}{\left(K+T k_{H}^{2}\right)^{2}}+\frac{\tilde{U}^{2}}{T^{2} k_{H}^{2}}\right) .
\end{aligned}
$$

Here $W$ is the energy dissipated per single superconducting layer, $L_{x}$ and $L_{y}$ are the dimensions of the crystal along the corresponding axis, and the coefficient 2 comes from the contribution from two $y z$ surfaces.

Keeping in mind the relation between electric field and velocity for the moving JV lattice $E_{z}=v H_{x} / c=\left(\Phi_{0} / 2 \pi c s\right) \omega$ and extracting parameters $\tilde{F}$ and $\tilde{U}$ from Eq. (28), we obtain for the excess conductivity

$$
\begin{aligned}
\sigma_{p}= & \left(\frac{2 \pi c s}{\Phi_{0}}\right)^{2} \frac{\eta n_{\xi}}{L_{x} s} \exp \left(-\frac{U_{\min }}{T}\right) \\
& \times \sqrt{\frac{2 \pi T}{a_{K} \varepsilon_{p}}} \frac{4 \lambda^{7}}{\pi^{2} \lambda_{J}^{4} h_{\|}^{2}} G\left[T / \varepsilon_{p}, h_{\|}\right],
\end{aligned}
$$

where

$$
G\left[\tilde{T}, h_{\|}\right]=\frac{\left[\mathcal{I}_{x}^{\prime}\left(h_{\|} u_{\min }\right)\right]^{2}}{\left(a_{K}+\tilde{T} h_{\|}^{2}\right)^{2}}+\frac{\mathcal{I}_{x}^{2}\left(h_{\|} u_{\min }\right)}{\tilde{T}^{2} h_{\|}^{4}},
$$

$u_{\text {min }} \approx \ln h$, and $\tilde{T}=T / \varepsilon_{p}$ is the dimensionless temperature. The pancake viscosity, $\eta$, also determines the flux-flow resistivity for the in-plane current, $\rho_{\mathrm{ff}}=s \Phi_{0} B /\left(c^{2} \eta\right)$. A natural scale of conductivity is the pancake flux-flow conductivity at field $B=\Phi_{0} /\left(4 \pi \lambda^{2}\right)$,

$$
\sigma_{\mathrm{ff}, \lambda}=\frac{4 \pi \lambda^{2} c^{2} \eta}{s \Phi_{0}^{2}}
$$

Using this scale, we can rewrite Eq. (31) in a somewhat more transparent form,

$$
\begin{aligned}
\sigma_{p}= & \sigma_{\mathrm{ff}, \lambda} \frac{\lambda}{\pi L_{x}} s^{2} n_{\xi} \exp \left(-\frac{U_{\mathrm{min}}}{T}\right) \\
& \times \sqrt{\frac{2 \pi T}{a_{K} \varepsilon_{p}}} \frac{\lambda^{4}}{\lambda_{J}^{4} h_{\|}^{2}} G\left[T / \varepsilon_{p}, h_{\|}\right] .
\end{aligned}
$$

In spite of the rather complicated general $\sigma_{p}\left(T, H_{x}, H_{z}\right)$ dependence (31), the main variation of $\sigma_{p}$ just below $H_{c 1}^{c}$ is related with the change of the concentration of the fluctuating pancakes. In samples with the lateral sizes larger than $\lambda$, this leads to the very characteristic exponential $\sigma_{p}$ dependence on $H_{z}$,

$$
\sigma_{p} \approx A\left(H_{x}, T\right) \exp \left(\frac{s \Phi_{0} H_{z}}{4 \pi T}\right),
$$

and the dominating temperature dependence of the prefactor is given by

$$
A\left(H_{x}, T\right)=\tilde{A}\left(H_{x}, T\right) \exp \left(-\frac{s \Phi_{0} H_{c 1}^{c}}{4 \pi T}\right) .
$$

These are the easiest qualitative predictions to compare with experiment. We remind the reader also that in small-size mesas, the expression in the exponent of Eq. (33) acquires a geometrical factor depending on mesa size, as described in Sec. II B 2.

A more complicated issue is the dependence of the excess conductivity on the in-plane magnetic field. The general formula (32) significantly simplifies at high in-plane fields $h_{\|} \gg 1$. In this regime, we obtain $G\left[\tilde{T}, h_{\|}\right] \approx 4 \pi^{2} /\left(\tilde{T}^{2} h_{\|}^{4}\right)$ and

$$
\sigma_{p} \approx \sigma_{\mathrm{ff}, \lambda} \sqrt{\frac{2 \pi}{a_{K}}} \frac{4 \pi \lambda}{L_{x}} s^{2} n_{\xi} \exp \left(-\frac{U_{\mathrm{min}}}{T}\right)\left(\frac{\varepsilon_{p}}{T}\right)^{3 / 2} \frac{\lambda^{4}}{\lambda_{J}^{4} h_{\|}^{6}},
$$

i.e., we expect that at high fields, the excess conductivity rapidly decreases with increasing in-plane field $\propto 1 / H_{x}^{6}$.

We have considered the influence of the fluctuating pancakes on the dense moving JV lattice only near the $y z$ surfaces, perpendicular to the in-plane magnetic field. Naturally, the fluctuating PV's also exist near the $x z$ surfaces and they also will contribute to dissipation. The JV lattice will displace them mainly along the $x$ axis. Accurate calculations for this case are more complicated because one has to take into account deformation of the JV lattice near the surface. However, even without performing the calculation of this contribution, it is evident that it will also be proportional to the PV's concentration, and therefore the dependence (33) will describe it as well. This dependence also should describe the behavior of the $c$-axis excess conductivity for smaller in-plane fields, in the regime of a dilute JV lattice. In this regime, the fluctuating PV's enhance dissipation of individual JV's, meaning that $\sigma_{p} \propto 1 / H_{x}$.

\section{EXPERIMENT}

\section{A. Experimental setup}

Bi2212 single crystals grown by the traveling solvent floating zone method were fabricated into mesas sandwiched by two superconducting electrodes of Bi2212 [see Fig. 1(a)] with a focused ion beam (FIB) machine SMI2050, SII Nanotechnology Inc. The fabrication details are described in Ref. 28. The studied samples listed in Table I include two medium-size mesas with lateral sizes $\gtrsim 5 \mu \mathrm{m}$ and four small mesas with lateral sizes $\sim 1 \mu \mathrm{m}$. Resistance of the mesa was

TABLE I. List of samples. $L, W$, and $t$ denote length $(\perp \boldsymbol{H})$, width $(\| \boldsymbol{H})$, and thickness $(\| c)$ of the mesa, respectively; see Fig. 1(a). $H_{p}$ is the JV flow oscillation period observed experimentally. Note that $H_{p}$ is slightly different from $\Phi_{0} / s L$ due to the ambiguity of the mesa dimensions. Units for dimensions, magnetic fields, and temperature are $\mu \mathrm{m}$, kOe, and $\mathrm{K}$, respectively.

\begin{tabular}{llclll}
\hline \hline & $L$ & $W$ & \multicolumn{1}{c}{$t$} & $H_{p}$ & $T_{c}$ \\
\hline Br0552 & 5.0 & 4.9 & 0.16 & 2.6 & 84.7 \\
Br0573 & 5.0 & 10.2 & 1.0 & 2.58 & 88.4 \\
Br0640 & 1.1 & 1.13 & 0.25 & 11.5 & 84 \\
Br0742 & 1.53 & 1.45 & 0.26 & 8.6 & 86 \\
Br0746 & 0.8 & 2.06 & 0.5 & 17.25 & 86.5 \\
Br0747 & 0.4 & 1.5 & 0.3 & 34.5 & 83.9 \\
\hline \hline
\end{tabular}


measured with the four-probe method with either dc or ac current excitation. For the ac excitation, the lock-in detection was used for voltage measurements. External magnetic fields $\boldsymbol{H}$ were applied by a split-pair superconducting magnet that generates a horizontal magnetic field up to $80 \mathrm{kOe}$, and the angle $\theta$ between the magnetic field and the $a b$ plane ( $x$ axis) was varied by rotating the sample probe with a precision rotator. The perpendicular ( $c$-axis) component of the magnetic field $H_{z}$ is given by $H \sin \theta$. In all plots presented here, $H_{z}$ is always swept from negative to positive, meaning that PV's are expelled from and introduced into the mesas in the negative and positive $H_{z}$ regions, respectively. Therefore, asymmetry with respect to the sign of $H_{z}$ in some plots may be caused by hysteretic behavior.

\section{B. Experimental results and discussion}

The $c$-axis resistivity $\rho_{c}$ in the vicinity of $\theta=0$ gives the JV flow resistance. With increasing $H_{z}, \rho_{c}$ suddenly drops due to penetrating PV's. Without taking fluctuating PV's into consideration, the value of the field when $\rho_{c}$ drops should correspond to the lower critical field $H_{c 1}$ (corrected by the demagnetization factor). The demagnetization factor of a small mesa ( $L$ or $W \simeq 1 \mu \mathrm{m}$ ) is small because the thickness of the whole crystal is about $10 \mu \mathrm{m}$ for all samples and it is larger than the width. For the midsize mesas ( $L$ or $W \simeq 5-10 \mu \mathrm{m}$ ), due to the demagnetization effect, the field at the edge of the junctions in the Meissner state should exceed the external field by a factor $\sim 1.2-1.3$. An example of $\rho_{c}$ as a function of $H_{z}$ at different applied fields for one of the medium-size mesas is plotted in Fig. 3(a). We see that the steep drop in $\rho_{c}$ in the low-field region is smeared with increasing parallel field $H$. We focus here on the quantitative characterization of behavior slightly below this drop.

Field behavior of small mesas has two important features. First, when the magnetic field is aligned with the layers, due to strong interaction with the edges, the dense JV lattice has a rectangular structure at almost all fields and only transforms into the triangular configuration in the vicinity of fields corresponding to an integer number of fluxes per junction, $H_{n}=n H_{p}$ with $H_{p}=\Phi_{0} / s L .{ }^{18,19}$ Values of $H_{p}$ for different mesas are listed in Table I. The $c$-axis resistivity has pronounced oscillations as a function of $H_{x}$ with maxima located near $H_{n} \cdot{ }^{11,12}$ Secondly, when the $c$-axis magnetic field is applied, penetration of the individual PV stacks can be resolved. In the case of magnetic-field tilting from the $a b$ plane, PV stacks penetrating into the mesa strongly impede JV flow along the $a b$ plane, which causes sharp drops in $\rho_{c}$. With increasing $H_{z}$, the separation between the drops decreases and finally corresponds to the period of one flux quantum per mesa area, $\Phi_{0} / L W$, as shown in Fig. 3(b). These quasiperiodic drops in $\rho_{c}$ are naturally attributed to penetrations of the individual PV stacks into the mesa. Even in the absence of JV's $(\boldsymbol{H} \| c)$, a stepwise increase in $\rho_{c}$ (shown as a solid line) indicated by arrows in Fig. 3(b) with a similar periodicity was observed. These steps are attributed to additional interlayer phase fluctuations caused by the penetrated PV stacks. ${ }^{28}$ Therefore, in small mesas, the $c$-axis resistivity has oscillating dependences on both field components.

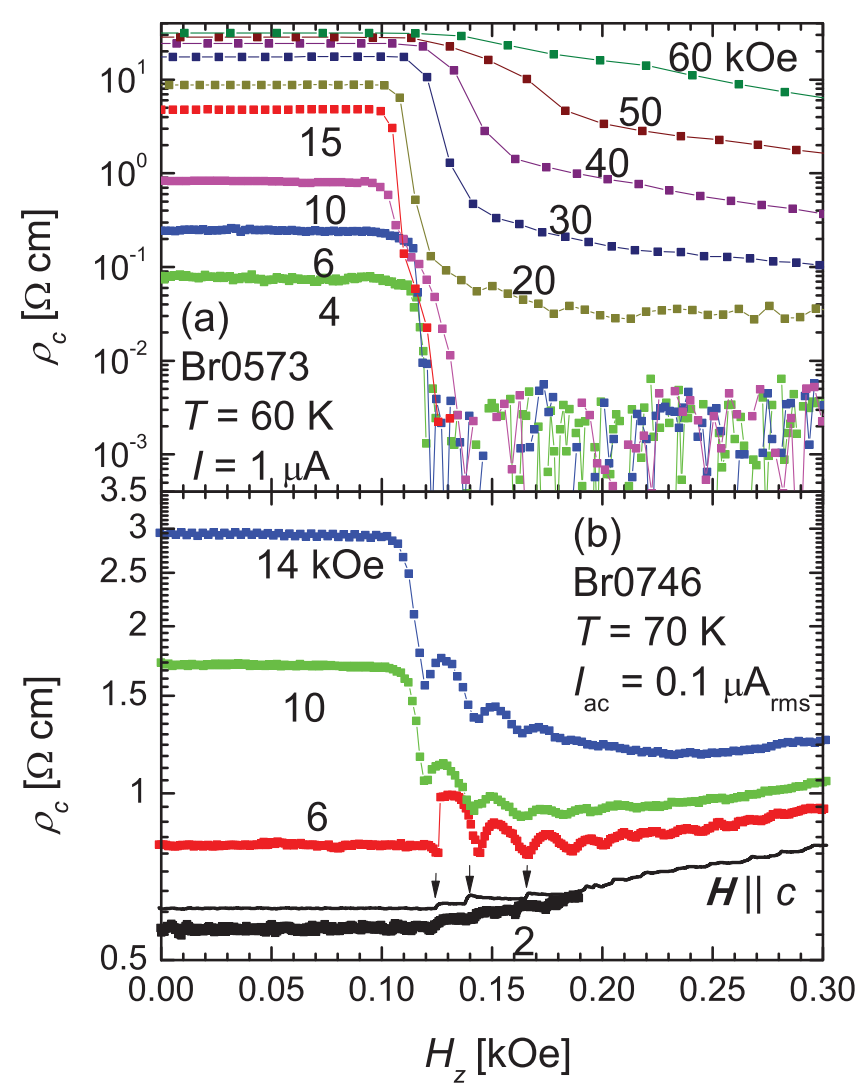

FIG. 3. (Color online) Typical dependences of the resistivity $\rho_{c}\left(H_{z}\right)$ in the midsize mesa $\mathrm{Br} 0573$ at $60 \mathrm{~K}$ (a) and small-size mesa Br0746 at $70 \mathrm{~K}$ (b). (a) Sharp drop corresponds to penetration of the PV stacks. We focus on the behavior below this drop. (b) In small-size mesas, in addition to the initial sharp drop, quasiperiodic drops due to penetration of the individual PV stacks are found. In the case of either small magnitude of tilting magnetic field $(2 \mathrm{kOe})$ or magnetic field parallel to the $c$ axis (solid line), stepwise changes in $\rho_{c}$ indicated by arrows are observed.

To quantify the additional dissipation of the JV lattice due to penetration of fluctuational PV's, we show the plots of the excess $c$-axis conductivity $\sigma_{p}\left(H_{x}, H_{z}\right)=\sigma_{c}\left(H_{x}, H_{z}\right)-$ $\sigma_{c}\left(H_{x}, 0\right)$ as a function of the $c$-axis magnetic field $H_{z}$ for several samples at different temperatures in the following figures. Here, $\sigma_{c}\left(H_{x}, 0\right)=1 / \rho_{c}\left(H_{x}, 0\right)$ is the conductivity for the magnetic field aligned with the layers at $\theta=0\left(H_{z}=0\right)$. Only the data at temperatures higher than $T=60 \mathrm{~K}$ are shown because at low temperatures (below $50 \mathrm{~K}$ ), the surface barrier and bulk pinning for PV's cause pronounced hysteresis in the dependences $\rho_{c}\left(H_{z}\right)$, which makes an analysis quite complicated.

Figure 4 illustrates the evolution of the $H_{z}$ dependence of the excess conductivity $\sigma_{p}$ with increasing field obtained from data presented in Fig. 3(a). Other mesas show similar behavior in the penetration region. In the figure, the predicted exponential dependence (33) is plotted as a thick solid line for each $T$. Since the constant $A$ is the sole fitting parameter, the slope of the semilog plot of the experimental data provides a direct test for Eq. (33). The first general observation is that the experimental data clearly show the existence of the exponential regime just before the penetration of the perpendicular field 


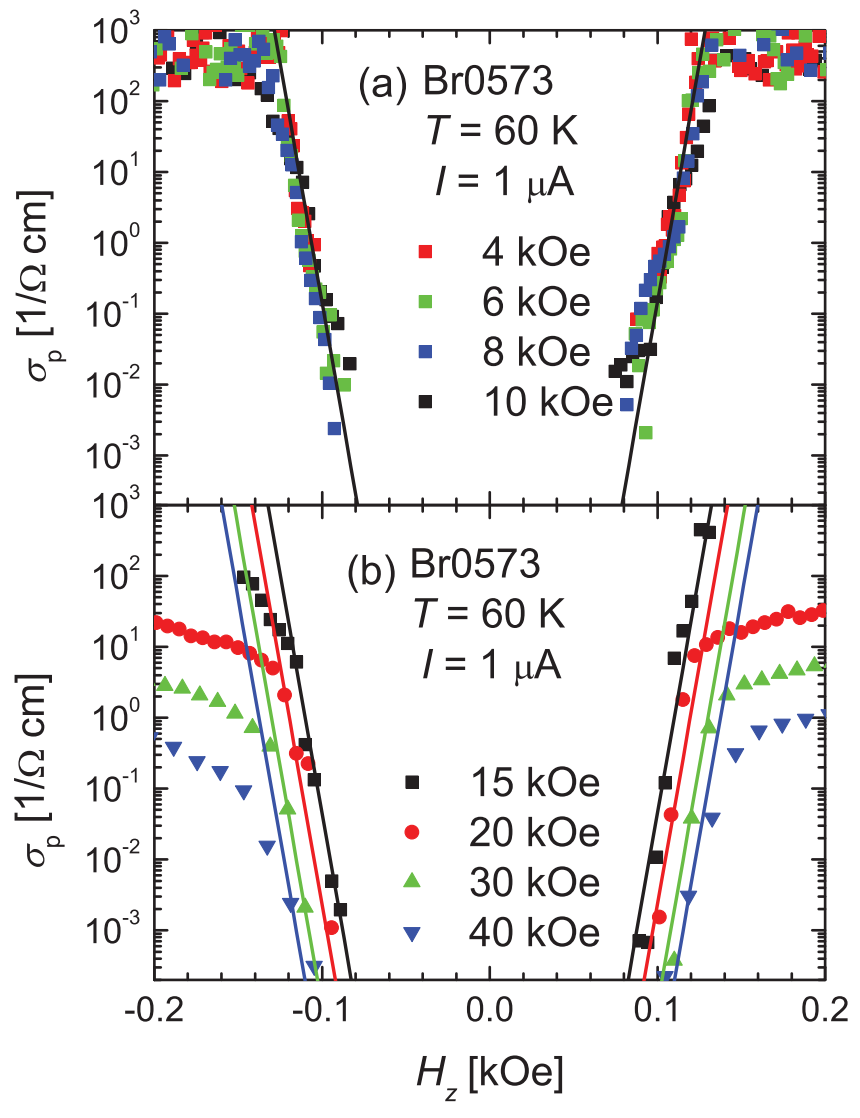

FIG. 4. (Color online) The dependences of the excess conductivity on $H_{z}$ for the sample $\mathrm{Br} 0573$ at $T=60 \mathrm{~K}$ in (a) $2<H<10$ kOe and (b) $15<H<60 \mathrm{kOe}$. Solid lines describe the exponential dependence (33). One can see that the data follow the theoretical prediction.

into the mesas. This exponential increase spans up to five orders of magnitude. The slopes of the $\ln \left(\sigma_{p}\right)$ versus $H_{z}$ curves are in good agreement with Eq. (33). The observation of this regime provides strong evidence of generation of the fluctuating PV near the sample surface.

There are several common features in the experimental data. The slopes of dependences $\ln \sigma_{p}$ versus $H_{z}$ remain independent of the in-plane field only up to the typical fields $\sim 40 \mathrm{kOe}$, in agreement with theory. At higher fields (not shown), the slope starts to decrease and at the same time the experimental dependencies $\sigma_{p}\left(H_{z}\right)$ are no longer symmetric over $\pm H_{z}$. At such a high magnetic field, it is probably difficult to reliably control the small $H_{z}$ component. At small fields $\lesssim 10 \mathrm{kOe}$, the excess conductivity is only weakly dependent on the field, while at higher fields it starts to decrease rapidly. This behavior is most probably related with the crossover into the dense-lattice regime. Figure 5 shows the dependence of the preexponential factor $A$ on the in-plane field $H_{x}$ for mesas Br0552 at $T=70 \mathrm{~K}$ and $\mathrm{Br} 0573$ at $60 \mathrm{~K}$. One can see that the decay at fields $H_{x}>6 \mathrm{kOe}$ is close to the theoretical $H_{x}^{-6}$ dependence given by Eq. (34).

\section{Small mesas: Triangular versus rectangular JV lattice}

An important feature of small-size mesas is the existence of regions of the rectangular vortex lattice at fields

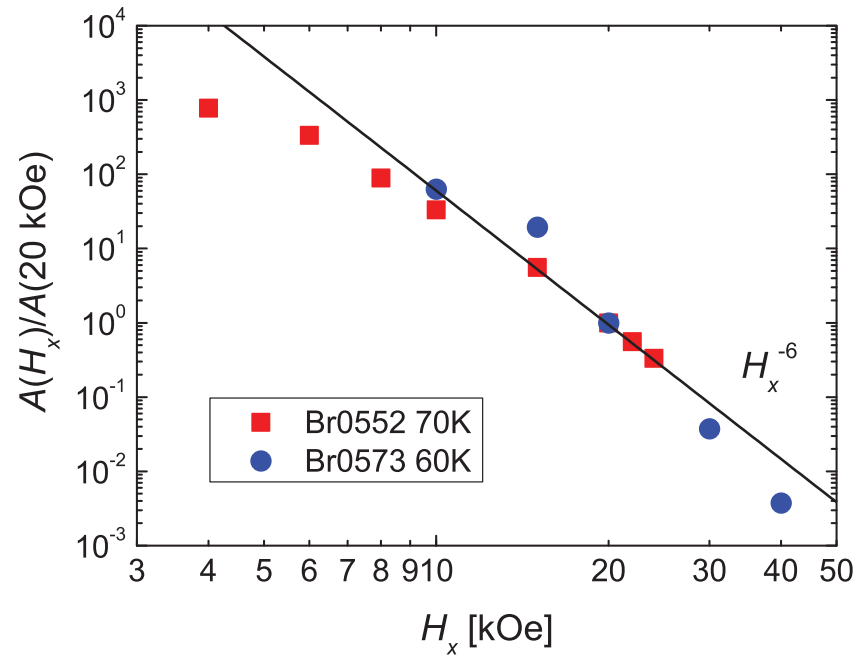

FIG. 5. (Color online) The normalized preexponential factor $A$ in Eq. (33) as a function of in-plane field $H_{x}$ for the mesa Br0552 at $70 \mathrm{~K}$ and $\mathrm{Br} 0573$ at $60 \mathrm{~K}$. The factor is normalized by its value at $H_{x}=20 \mathrm{kOe}$.

$H_{x}>B_{\mathrm{cr}} L /(\gamma s)$. In this range with increasing magnetic field, the JV lattice undergoes a series of structural phase transitions between the triangular and rectangular configurations, with triangular structures located around the fields $n H_{p}$ and rectangular structure located around the fields $(n+1 / 2) H_{p}$. The widths of the triangular regions shrink with increasing field. ${ }^{18,19}$ The triangular configuration is expected to have a much stronger interaction with the PV's than the rectangular lattice because there are no in-plane currents in the latter state.

Figure 6 presents the $H_{z}$ dependences of the excess conductivity for two small-size mesas Br0746 and Br0742. The panel (a) shows data for mesa $\mathrm{Br} 0746$ at $75 \mathrm{~K}$ for fields smaller than $H_{p}=17.26 \mathrm{kOe}$. We can see that there is again a wide range of exponential dependence and the slope $d \ln \sigma_{p} / d H_{z}$ agrees with theoretical predictions. Figure 6(b) shows the excess conductivity for the small mesa $\mathrm{Br} 0742$ for three values of field 4.3, 8.6, and $12.8 \mathrm{kOe}$ almost corresponding to 0.5 , 1.0 , and $1.5 H_{p}$. At the last field, the rectangular lattice is realized. We can see that while agrement with the predicted dependence is very good for the first two values of the field, for the last field the excess conductivity before penetration is much smaller, which makes the transition much sharper. This confirms that the fluctuating pancakes have a much weaker influence on dissipation of the rectangular vortex lattice.

We finish this subsection with a small comment on the oscillating behavior of the $c$-axis resistivity in small-size mesas at larger $H_{z}$ due to the penetrating individual PV stacks illustrated in Fig. 3(b). This oscillating behavior is a result of the competition between the two opposite factors. On the one hand, the PV's in the stacks directly contribute to the dissipation of the JV lattice reducing the resistivity. On the other hand, penetration of the magnetic flux inside the mesa relaxes the Meissner currents near its surface, which reduces the contribution coming from the fluctuating PV's and increases the resistivity. We can see that slightly above the penetration field the resistivity increases, meaning that the second mechanism actually prevails in this region. Moreover, 


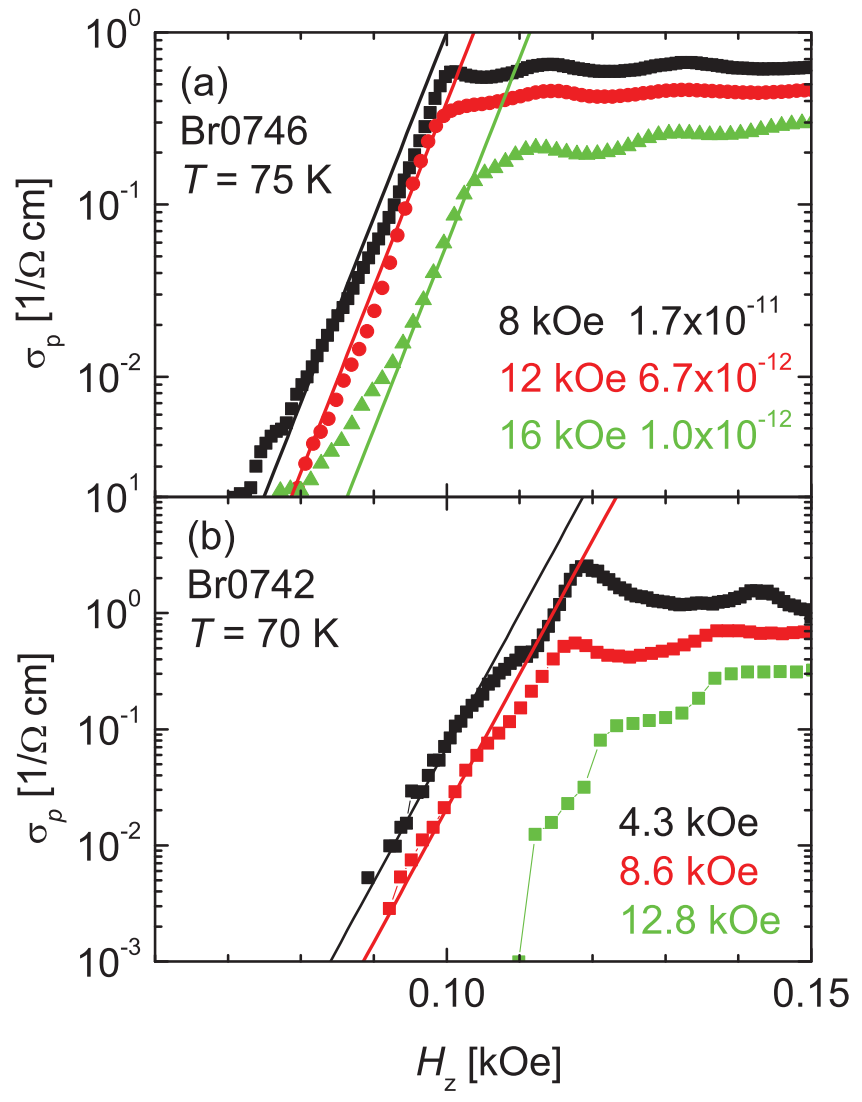

FIG. 6. (Color online) The excess pancake conductivity for two small-size mesas. (a) Data for the mesa Br0746 at $75 \mathrm{~K}$ for three values of magnetic field smaller than $H_{p}=17.25 \mathrm{kOe}$. Theoretical exponential dependences and corresponding preexponential factors are also shown. (b) The excess conductivity for the sample $\mathrm{Br} 0742$ at $70 \mathrm{~K}$ also for three values of field. For the first two values 4.3 and $8.6 \mathrm{kOe}$ corresponding to $0.5 H_{p}$ and $1.0 H_{p}$, agreement with theory is very good. There is no agreement for the last value, $12.8 \mathrm{kOe} \approx$ $1.5 H_{p}$; the transition is much sharper and occurs at higher $H_{z}$. At this value, the rectangular JV lattice is realized in the mesa, which is less sensitive to the fluctuating PV's.

the continuous increase of the resistivity for $H=10$ and $14 \mathrm{kOe}$ indicates that the full PV stack in the mesa center is not formed at once but continuously builds within the finite field range.

\section{Evolution with increasing temperature and finite-size effect in small mesas}

Figure 7 presents the evolution of the $\sigma_{p}$ versus $H_{z}$ dependences with increasing temperature at fixed field for two small-size mesas. In both cases, the fields are close to the corresponding values of $H_{p}$. We can see that for the mesa Br0640 [panel (a)], these dependences agree with the prediction for the large-size regime for temperatures 60,70 , and $75 \mathrm{~K}$, but the slope $d \ln \sigma_{p} / d H_{z}$ becomes considerably smaller for $80 \mathrm{~K}$. For the smallest mesa Br0747 with the width $L=0.4 \mu \mathrm{m}$ [panel (b)], the slopes are smaller than predicted by Eq. (33) for all temperatures and decrease with increasing temperature. This behavior can be naturally explained by the finite-size effect considered in Sec. II B 2. As the other size
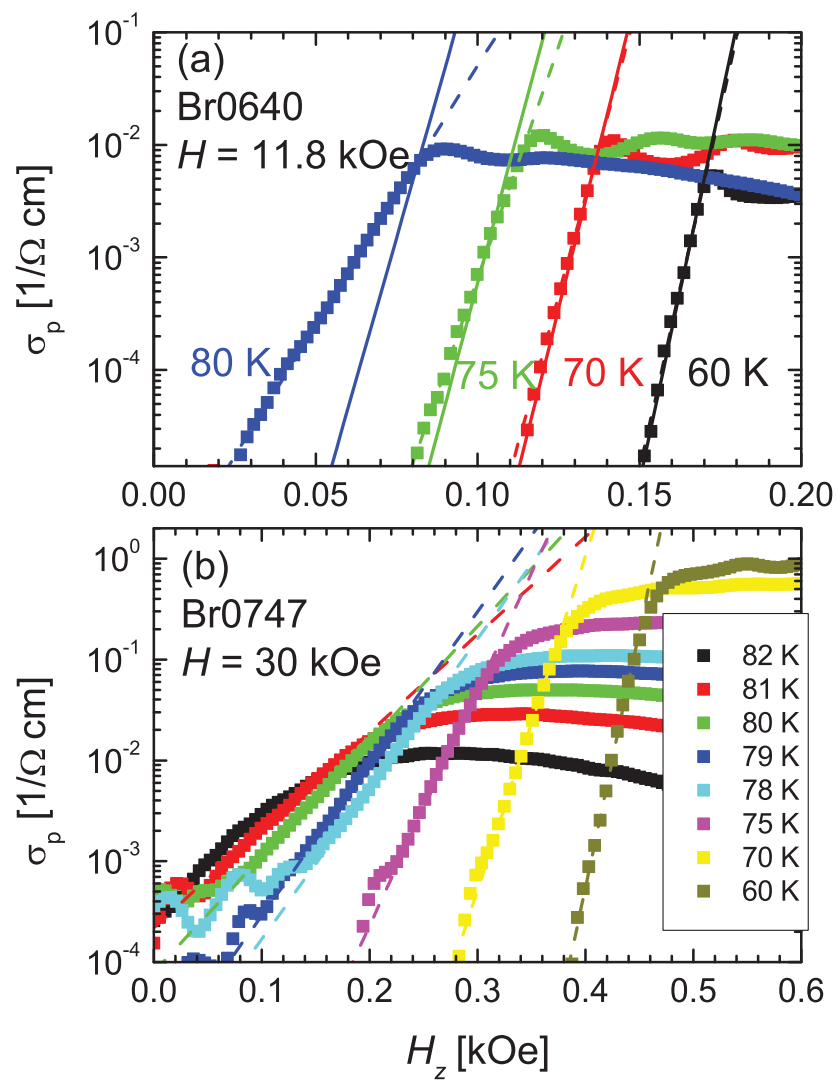

FIG. 7. (Color online) Temperature evolution of the excess conductivity for small-size mesas. (a) Excess-conductivity plots for the small-size mesa Br0640 for the field $11.8 \mathrm{kOe}$ corresponding to the triangular lattice at four values of temperature, 60, 70, 75, and 80 K. Solid lines represent exponential dependences given by Eq. (33) at temperatures for corresponding colors, whereas the broken lines are given by Eq. (35) for the small-size regime. One can see that the exponential dependencies agree with the prediction for the large-size regime at 60,70 , and $75 \mathrm{~K}$, but the slope is considerably smaller at $80 \mathrm{~K}$. (b) Data for the mesa Br0747 from 60 to $82 \mathrm{~K}$ at $3 \mathrm{~T}$. The exponential dependence for the small-size regime (35) shown by broken lines describes the data very well.

of this mesa $W$ is 3.75 times larger than the width $L$, we can apply the strip approximation. In this case when the London penetration depth $\lambda$ becomes comparable with the mesa's smallest lateral size, the energy minimum of the $\mathrm{PV}, U_{\min }$, is shifted to its center and the slope of $U_{\min }$ versus $H_{z}$ decreases, thus $H_{z}$ must be substituted by $H_{z}\left[1-\cosh ^{-1}(L / 2 \lambda)\right]$ :

$$
\sigma_{p} \approx A\left(H_{x}, T\right) \exp \left\{\frac{s \Phi_{0} H_{z}}{4 \pi T}\left[1-\cosh ^{-1}\left(\frac{L}{2 \lambda}\right)\right]\right\} .
$$

This means that from the slopes we can extract the temperaturedependent $\lambda$. Figure 8(a) presents the temperature dependence of $\lambda^{-2}$ obtained in such a way. It shows an expected linear dependence near $T_{c}, \lambda^{-2}=\lambda_{0}^{-2}\left(1-T / T_{c}^{\mathrm{MF}}\right)$, with the Ginzburg-Landau value $\lambda_{0}=0.1 \mu \mathrm{m}$ and the mean-field transition temperature $T_{c}^{\mathrm{MF}} \approx 86 \mathrm{~K}$, which is $2 \mathrm{~K}$ higher than the resistivity transition temperature for this mesa, $T_{c}=84 \mathrm{~K}$. Additional information can be obtained from the temperature dependence of the preexponential factor $A$ presented in the right part of Fig. 8. As follows from Eq. (18), it has an 

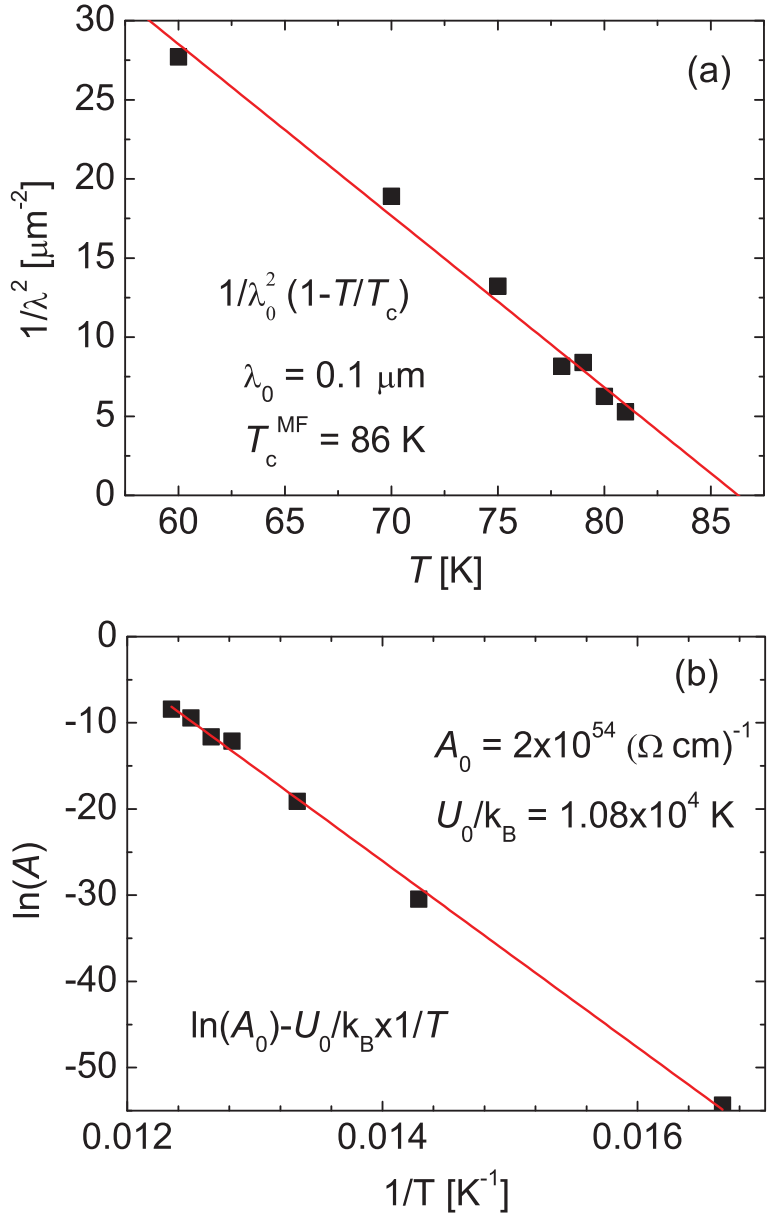

FIG. 8. (Color online) (a) Temperature dependence of $\lambda^{-2}$ extracted from slopes $d \ln \sigma_{p} / d H_{z}$ for mesa $\mathrm{Br} 0747$ using the activation energy, Eq. (18). (b) Arrhenius plot of the preexponential factor $A$ for the same mesa. The origin of the huge value of $A_{0}$ is explained in the text.

Arrhenius temperature dependence, $A=A_{0} \exp \left(-U_{0} / k_{B} T\right)$, with the activation energy $U_{0}=\left[s \Phi_{0}^{2} /\left(4 \pi \lambda_{0}\right)^{2}\right][\ln (L / \pi \xi)+$ $0.5]$. This agrees with experiment and the fit gives $U_{0} / k_{B}=$ $1.08 \times 10^{4} \mathrm{~K}$. This is consistent with the above value of $\lambda_{0}$ if we assume $\xi \approx 50 \mathrm{~nm}$ at $75 \mathrm{~K}$. Note also that the huge value of $A_{0}$ obtained from the fit, $A_{0} \approx 2 \times 10^{54}(\Omega \mathrm{cm})^{-1}$, is, in fact, very reasonable because $A_{0}$ contains the factor $\exp \left(U_{0} / k_{B} T_{c}^{\mathrm{MF}}\right) \approx \exp (126) \approx 5 \times 10^{54}$. The obtained value of $\lambda_{0}$ for our overdoped small mesa, however, is somewhat smaller than the values reported in the literature. ${ }^{29}$ Note that the mesa Br0640 also shows the same trend. However, for this mesa the sizes $W$ and $L$ are close, so that the narrow strip model is not applicable. An accurate description of this case requires numerical analysis.

\section{CONCLUSIONS}

Layered superconductors in magnetic fields are characterized by complex interplay between the Josephson and pancake vortices. In particular, dissipation of the Josephson vortex lattice is proven to be extremely sensitive to the presence of pancake vortices. We utilize this property to probe the fluctuating pancake vortices. In the Meissner state, in the $c$-axis magnetic field smaller than the lower critical field, the pancake vortices cannot form the Abrikosov vortex lines. In the fluctuational regime, however, the individual pancake vortices may exist near the surface or inside small samples. They lead to additional contribution to the $c$-axis conductivity of the Josephson vortex lattice, which has a very characteristic exponential dependence on the $c$-axis magnetic field. While in mesas with lateral sizes significantly larger than the London penetration depth $\lambda$ the slope $d \ln \left(\sigma_{p}\right) / d H_{z}$ of this dependence is universal, in smaller mesas it acquires the geometrical factor depending on the ratio size/ $\lambda$. We systematically studied the excess $c$-axis conductivity of the JV lattice due to the fluctuating pancake vortices in mesas fabricated out of $\mathrm{Bi}_{2} \mathrm{Sr}_{2} \mathrm{CaCu}_{2} \mathrm{O}_{8+\delta}$ crystals. The predicted exponential dependence is clearly observed and its slope agrees very well with the theoretical value. Analyzing the temperature evolution of the slope in the mesa with the smallest width and extracting the geometrical factor, we were able to restore the temperature dependence of the London penetration depth. These findings provide strong evidence for the existence of the fluctuating pancakes in the Meissner state and demonstrate that the Josephson-vortex lattice provides a unique tool to probe them.

\section{ACKNOWLEDGMENTS}

A.E.K. was supported by UChicago Argonne, LLC, operator of Argonne National Laboratory, a US Department of Energy Office of Science laboratory, operated under Contract No. DE-AC02-06CH11357. A.I.B. also would like to acknowledge support from Argonne National Laboratory for the one-month visit during which this work was initiated. I.K. was supported by a Global COE program on photonics and electronics science and engineering at Kyoto University, Kansai Research Foundation, and Mazda Foundation.
*Present address: Quantum Beam Science Directorate, Japan Atomic Energy Agency, Takasaki, Gunma 370-1292, Japan.

${ }^{1}$ L. N. Bulaevskii and J. R. Clem, Phys. Rev. B 44, 10234 (1991).

${ }^{2}$ K. Efetov, Sov. Phys. JETP 49, 905 (1979); A. I. Buzdin and D. Feinberg, J. Phys. 51, 1971 (1990); S. N. Artemenko and A. N. Kruglov, Phys. Lett. A 143, 485 (1990); J. R. Clem, Phys. Rev. B 43, 7837 (1991).

${ }^{3}$ A. E. Koshelev, Phys. Rev. Lett. 83, 187 (1999).
${ }^{4}$ I. V. Grigorieva, J. W. Steeds, G. Balakrishnan, and D. M. Paul, Phys. Rev. B 51, 3765 (1995); C. A. Bolle, P. L. Gammel, D. G. Grier, C. A. Murray, D. J. Bishop, D. B. Mitzi, and A. Kapitulnik, Phys. Rev. Lett. 66, 112 (1991); A. Tonomura, H. Kasai, O. Kamimura, T. Matsuda, K. Harada, T. Yoshida, T. Akashi, J. Shimoyama, K. Kishio, T. Hanaguri, K. Kitazawa, T. Masui, S. Tajima, N. Koshizuka, P. L. Gammel, D. Bishop, M. Sasase, and S. Okayasu, ibid. 88, 237001 (2002); A. E. Koshelev, Phys. Rev. B 71, 174507 (2005). 
${ }^{5}$ A. Grigorenko, S. Bending, T. Tamegai, S. Ooi, and M. Henini, Nature (London) 414, 728 (2001).

${ }^{6}$ S. J. Bending and M. J. W. Dodgson, J. Phys. Condens. Matter 17, R955 (2005).

${ }^{7}$ J. U. Lee, J. E. Nordman, and G. Hohenwarter, Appl. Phys. Lett. 67, 1471 (1995); J. U. Lee, P. Guptasarma, D. Hornbaker, A. El-Kortas, D. Hinks, and K. E. Gray, ibid. 71, 1412 (1997).

${ }^{8}$ G. Hechtfischer, R. Kleiner, A. V. Ustinov, and P. Müller, Phys. Rev. Lett. 79, 1365 (1997); G. Hechtfischer, R. Kleiner, K. Schlenga, W. Walkenhorst, P. Müller, and H. L. Johnson, Phys. Rev. B 55, 14638 (1997).

${ }^{9}$ Yu. I. Latyshev, P. Monceau, and V. N. Pavlenko, Physica C 282287, 387 (1997); 293, 174 (1997); Yu. I. Latyshev, M. B. Gaifullin, T. Yamashita, M. Machida, and Y. Matsuda, Phys. Rev. Lett. 87, 247007 (2001).

${ }^{10}$ S. Ooi, T. Mochiku, and K. Hirata, Phys. Rev. Lett. 89, 247002 (2002).

${ }^{11}$ I. Kakeya, M. Iwase, T. Yamamoto, and K. Kadowaki, e-print arXiv:cond-mat/0503498; S. Urayama, T. Hatano, H. B. Wang, M. Nagao, S. M. Kim, and J. Arai, e-print arXiv:cond-mat/0602659.

${ }^{12}$ I. Kakeya, Y. Kubo, M. Kohri, M. Iwase, T. Yamamoto, and K. Kadowaki, Phys. Rev. B 79, 212503 (2009).

${ }^{13}$ A. E. Koshelev, Yu. I. Latyshev, and M. Konczykowski, Phys. Rev. B 74, 104509 (2006); Yu. I. Latyshev, M. Konczykowski, and S.-J. Kim, JETP Lett. 89, 342 (2009).

${ }^{14}$ G.-H. Lee, Y.-D. Jin, and H.-J. Lee, Phys. Rev. B 81, 174508 (2010)
${ }^{15}$ Note that we consider here the superconducting mesas with low demagnetization factor along $H_{z}$.

${ }^{16}$ A. Buzdin and D. Feinberg, Phys. Lett. A 167, 89 (1992).

${ }^{17}$ R. G. Mints, I. B. Snapiro, and E. H. Brandt, Phys. Rev. B 54, 9458 (1996).

${ }^{18}$ M. Machida, Phys. Rev. Lett. 96, 097002 (2006).

${ }^{19}$ A. E. Koshelev, Phys. Rev. B 75, 214513 (2007).

${ }^{20} \mathrm{The}$ particle current $\mathbf{j}$ in this section describes flow of the pancake vortices proportional to the local electric field and should not be confused with the electric current.

${ }^{21}$ We measure the temperature $T$ in the energy units throughout the paper. Conversion to kelvins can be made by the usual substitution $T \rightarrow k_{B} T$, where $k_{B}$ is the Boltzmann constant.

${ }^{22}$ V. N. Kopylov, A. E. Koshelev, I. F. Schegolev, and T. G. Togonidze, Physica C 170, 291 (1990); L. Burlachkov, V. B. Geshkenbein, A. E. Koshelev, A. I. Larkin, and V. M. Vinokur, Phys. Rev. B 50, 16770 (1994).

${ }^{23}$ V. G. Kogan, Phys. Rev. B 75, 064514 (2007).

${ }^{24}$ A. A. Abrikosov, Fundamentals of Theory of Metals (NorthHolland, Amsterdam, 1988).

${ }^{25}$ A. Buzdin and J. P. Brison, Phys. Lett. A 196, 267 (1994).

${ }^{26}$ G. Böbel, Nuovo Cimento 38, 1740 (1965).

${ }^{27}$ L. N. Bulaevskii, M. Maley, H. Safar, and D. Dominguez, Phys. Rev. B 53, 6634 (1996).

${ }^{28}$ I. Kakeya, K. Fukui, K. Kawamata, T. Yamamoto, and K. Kadowaki, Physica C 468, 669 (2008).

${ }^{29}$ W. Anukool, S. Barakat, C. Panagopoulos, and J. R. Cooper, Phys. Rev. B 80, 024516 (2009). 